Resumen: El propósito de este escrito consiste en aportar algunas precisiones en torno a una posible epistemología del diseño, reflexionando sobre los distintos enfoques que puede asumir una investigación en el campo del proyecto

Palabras clave: Epistemología del Diseño - investigación sobre el Diseño - investigación para el Diseño - investigación a través del Diseño - investigación proyectual.

[Resúmenes en inglés y portugués en la página 53]

(1) Ana Cravino. Doctora FADU-UBA. Magister en Gestión de Proyectos educativos, CAECE. Profesora Superior Universitaria, UM. Arquitecta UM. Profesora de Laboratorio 2 (Taller de Tesis) en el Doctorado en Diseño, Universidad de Palermo, desde 2014. Investigadora principal del Instituto de Arte Americano e Investigaciones Estéticas "Mario Buschiazzo", FADU-UBA, 2015. Investigadora de las Universidad de Buenos Aires, Palermo y del ITBA Investigadora categorizada del Programa de Incentivos docentes del Ministerio de Educación de la Nación. Profesora invitada en universidades de Ecuador, México y Colombia. Autora de varios libros y artículos científicos en revistas nacionales e internacionales. 


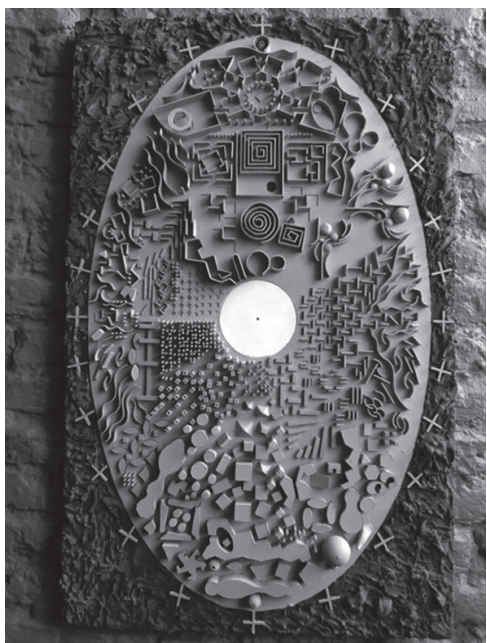

Cartografía de espacialidades (Jorge Pokropek)

\section{Precisiones preliminares}

Con este título queremos expresar dos cuestiones: Una, la ineludible tarea que es necesario emprender si es que deseamos construir teorías validadas en el campo del Diseño, por lo que este trabajo pretende ser un aporte, aunque sea pequeño, en el sendero de establecer una epistemología que formule criterios para juzgar las teorías propuestas. La segunda cuestión, se refiere a la indispensable apertura que debe poseer esta epistemología, debida cuenta lo complejo de la disciplina cuyos límites borrosos se expanden, lo cual nos lleva a sugerir que nuestras reflexiones se encaminan a elaborar "una" y no "la" epistemología del diseño entre muchas miradas convergentes que se puedan plantear.

Coincidimos, entonces, con Nigel Cross (2001) respecto a que el Diseño posee una cultura propia, intelectual y práctica, que constituye una base fundamental para la enseñanza, diferente de la de las Ciencias Naturales, las Artes y las Humanidades.

Coincidimos también con Roberto Doberti (2006) en referencia a que las prácticas proyectuales constituyen una cuarta posición diferente al arte, la tecnología y la ciencia. Por consiguiente no es posible transpolar una definición pensada para otro tipo de saberes. Esto nos lleva a sostener la existencia de un tipo peculiar de conocimiento que es el proyectual.

Y es por eso que también coincidimos con Sarquis (2004) respecto a la producción poiética de conocimiento. Recordemos que Platón (1871) define en El Banquete al término poiesis como "la causa que convierte cualquier cosa que consideremos de no-ser a ser" (p. 341 ), entendiendo por poiesis todo proceso creativo, mientras que Aristóteles diferencia la poiesis de la praxis, pues en esta última, la finalidad de la acción es la propia acción que se lleva a cabo. 
Sin embargo, vale aclarar que no todo el conocimiento empleado en el proyecto surge de una práctica proyectual. En este sentido Christopher Frayling (1993), en un breve pero sustancioso artículo, realiza una distinción entre los tipos de investigación que se llevan a cabo en el campo del arte y del diseño, que propone unificados, diferenciando entre "investigación sobre el arte", "investigación para el arte" e "investigación a través del arte".

El primer tipo, que toma como objeto de estudio a los productos artísticos y de diseño, no se diferencia demasiado de la reflexión sobre otras elaboraciones culturales, aunque el enfoque elegido puede ser histórico, antropológico, sociológico, semiótico, estético, e incluso, económico. Es claro en este enfoque de investigación el distanciamiento con el objeto de estudio, siendo prioritaria la mirada desde el marco teórico de la disciplina elegida para realizar el análisis. El modelo fundacional de esta indagación sería el hermenéutico, empleado por la crítica de arte. Sin embargo, es necesario que el objeto de estudio disciplinar no quede minimizado frente al aparato teórico y metodológico extradisciplinar. Y también es necesario que la herramienta conceptual sea pertinente con el objeto y consistente en sus planteos. En este mismo sendero, afirma Roberto Fernández (2013, p.19) que existe cierta tendencia actual que presupone, por ejemplo, para la investigación en arquitectura la adquisición de:

... cierto estatus de cientificidad en tanto se asimile, como una suerte de subproducto, a campos desarrollados por otras disciplinas, como los estudios de arquitectura tributarios de la ciencia histórica o los estudios urbanísticos insertos en temas y métodos de la ciencia geográfica...

El segundo tipo tampoco ofrece muchos obstáculos para su comprensión, ya que analiza al diseño desde las ciencias auxiliares, es decir, recurriendo a todo el conocimiento que se necesita para el diseño. De esta manera, el mencionado enfoque puede apelar al análisis de aspectos contextuales, ergonómico-funcionales, tecnológicos, materiales, ambientales, socio-culturales, etc. Mucho del conocimiento que se emplea y se obtiene nutre a las asignaturas denominadas "de apoyo" o "complementarias" que poseen los distintos planes de estudio de las diversas carreras proyectuales respecto al cual los futuros profesionales reciben entrenamiento. Según HenkBorgdorff (2010, p.9):

...puede describirse como una investigación aplicada, en sentido estricto. En este tipo, el arte no es tanto el objeto de investigación, sino su objetivo. La investigación aporta descubrimientos e instrumentos que tienen que encontrar su camino hasta prácticas concretas de una manera u otra.

La investigación sobre sistemas de información geográfica, mercadeo, características del usuario, comitente o contexto socioespacial, materiales de construcción, modos de estampado, técnicas de filmación,programas de dibujo o, por ejemplo, la indagación sobre una patología médica que requiera un tipo especial de diseño de prótesis, serían muestras equiparables a la investigación para el diseño, la arquitectura o el ur- 
banismo. Este tipo de búsqueda se realiza habitualmente de manera previa al desarrollo del Proyecto tanto por estudiantes como por profesionales. Como su finalidad es ser útil al Proyecto, no cumple las pautas de escritura de una investigación académica, de modo que, muchas veces, simplemente recopila datos y, a lo sumo, los interpreta o los procesa sistemáticamente.

El primer tipo de investigación es asimilable, por su carácter cualitativo, al de las ciencias sociales. Mientras que el segundo se asemeja más al modelo de las ciencias naturales por su modalidad cuantitativa o cuanti-cuali, aunque frecuentemente no avanza más allá del dato y no tiene pretensión de obtener un conocimiento generalizable. El tercer tipo, al que Frayling (1993) llama investigación “a través del arte” y Borgdorff (2010) "en el arte", es aquel que obtiene conocimiento disciplinar durante y por medio de la práctica proyectual. Es el más controvertido de los tres, y es el que más claramente define el conocimiento disciplinar. Sobre esta clase de investigación se sitúan las definiciones de Sarquis, Doberti y Cross.

\section{Irrealismo Filosófico}

Nelson Goodman (1995) nos desconcierta con su constructivismo irrealista al afirmar explícitamente que "lo que hay consiste en lo que hacemos" (p. 57) y que el mundo acaba disolviéndose en sus versiones.

Su definición de lo artefactual rompe con la taxonomía de Simon (1996), quien estipula a las ciencias de lo artificial como aquellas que se ocuparían de lo no natural, presuponiendo, por oposición aquella última categoría como algo preexistente, obvio. Recordemos, entonces, la tradicional clasificación de las ciencias. Nos encontramos, por un lado, con una primera división entre formales y fácticas, siendo las primeras la matemática y la lógica, mientras que, por el otro, dentro de la segunda clasificación se encontrarían todas las ciencias empíricas: tanto las naturales y como las sociales. Pero para Goodman no hay lugar para lo natural puesto que:

Lo que es más importante, no podemos encontrar ningún rasgo-del- mundo que sea independiente de todas las versiones. Todo lo que se puede decir con la verdad de un mundo depende de la acción de decir -no del hecho de lo que digamos sea verdadero, sino de lo que decimos con verdad (o bien aparece como correcto) participa de y es relativo, no obstante, a un lenguaje o a otro sistema de símbolos que utilicemos-. No se puede trazar ninguna frontera clara entre los rasgos-del-mundo que depende del discurso y aquellos que no dependen de él. Como he dicho, "en la práctica, por supuesto, trazamos la frontera donde queremos y la cambiamos tan a menudo como convenga a nuestros propósitos" (p.74).

No hay clases naturales que existan dadas de antemano en un mundo por fuera de nosotros e independientemente, a la espera que las descubramos, sino que hay clases 
relevantes que forman parte de nuestras versiones de mundo las cuales obedecen a nuestros intereses.

Ian Hacking va más allá, cuestiona con Dewey el dualismo entre pensar y hacer, y rechaza el primer Wittgenstein que establecía una teoría representacional de la verdad en el sentido que pensamiento, lenguaje y mundo deberían tener la misma forma, puesto que una oración verdadera solo era una representación o copia del mundo. Por el contrario, para Hacking (1996):

La primera invención característicamente humana es la representación. Una vez que hay una práctica a representar, viene inmediatamente a continuación un concepto de segundo orden. Este es el concepto de la realidad, un concepto que tiene contenido solo cuando hay representaciones de primer orden (p. 163).

El constructivismo de Goodman y Hacking ponen sobre el tapete que no trabajamos con datos del mundo sino con construcciones fenoménicas producto de dinámicas intencionales y simbólicas. Y en este sentido es tan artificial una teoría científica como un afiche o una silla.

Recuperemos una definición de Adolfo Corona Martínez (1990), quien afirma que:

El diseñador inventa el objeto en el acto mismo de representarlo; esto es, dibuja un objeto inexistente, cada vez con mayor precisión. Esta precisión es un aumento en el detalle, dentro del sistema de reglas de la representación misma. Así el diseño es la descripción progresiva de un objeto que no existe al comenzar la descripción (p.39).

Al igual que Hacking, Corona Martínez estipula primero la existencia de la representación y luego, a consecuencia de ello, la presencia del mundo. Versiones y mundo se entremezclan.

Agreguemos a este contexto el llamado giro pragmático de la filosofía, el cuál introduce un tercer componente a la clásica relación de conocimiento sujeto-objeto y este elemento es la esfera de la práctica. Por ende el conocimiento ya no puede ser entendido como una simple representación de lo real sino como una construcción, cuyas reglas se definen por medio de prácticas intersubjetivasen función de intereses y objetivos (Cabanchik et al, 2003). El límite entre teoría y práctica se desdibuja, así como el papel subordinado de esta última. De modo que el diseño, y su posible epistemología, no constituyen un aparato filosófico menor o parasitario de otros saberes, sino que podría audazmente presentarse como un modelo genuino para otras investigaciones.

\section{Investigación proyectual}

Es sabido que los procesos proyectuales son productores de conocimiento (Martín Iglesia et alt, 2012) (Cravino, 2020) (Fernández, 2013). En este sentido Donald Schon (1998), apo- 
yándose en el enfoque pragmatista de Dewey y en el constructivismo de Goodman, sostiene que "La conversación reflexiva que un diseñador establece con los materiales de una determinada situación puede generar nuevos descubrimientos, significados e invenciones" (p.50). Es por ello que Schon propone para el diseño el modelo de investigación acción, donde la teoría y la práctica aparecen imbrincadas. Este tipo de investigación reconoce los límites de la racionalidad técnica y no elude la incertidumbre.

Sin embargo, el conocimiento proyectual no equivale necesariamente a investigación. En toda práctica de diseño hay conocimiento, pero para que haya una investigación genuina tiene que haber una intencionalidad, puesto que toda investigación debe contar con una argumentación sólida y una evidencia empírica que brinden conjuntamente el apoyo teórico y práctico que necesita una hipótesis para ser validada.

$\mathrm{Y}$ una hipótesis es un acto creativo que responde a un problema construido. $\mathrm{Y}$ en este punto no hay diferencias con otros campos de saber.

Una investigación a través del proyecto se asemeja más a una investigación tecnológica que a una científica. Mientras que la ciencia engendra saber, el diseño y la tecnología comparten la generación de un doble producto: el conocimiento y el artefacto. Ambos, conocimiento y artefactos son lo que hay y son lo que hacemos.

Indagar sobre el proceso poético de creación de artefactos y sobre los propios artefactos produce conocimiento. $\mathrm{Y}$ este conocimiento define a la disciplina y a nosotros mismos.

\section{Bibliografía}

Borgdorff, H. (2010).El debate sobre la investigación en las artes En Cairon: revista de ciencias de la danza No 13 , pp. 47-66

Cabanchik, S.; Penelas, F.; Tozzi, V.(2003) (comp.) El giro pragmático en la filosofía. Barcelona:Gedisa

Corona Martinez, A. (1990). Ensayo sobre el Proyecto. Buenos Aires: Editorial CP67

Cravino, Ana (2020) Hacia una Epistemología del diseño En Cuadernos del Centro de Estudios en Diseño y Comunicación No82, agosto 2020, pp. 33-45

Cross, N. (2001). "Designerly Ways of Knowing: Design Discipline Versus Design Science". EnDesignIssues Vol 17, No 3, Summer 2001, MIT. http://users.metu.edu.tr/baykan/ arch467/Readings/Cross01.pdf

Cross, N. (1993). A History of Design Methodology en de Vries, M. J.; Cross, N. \& Grant, D. P. (1993).Design Methodology and Relationships with Science, Springer Science \& Business Media, Kluwer

Academic Publishers, Dordrecht, pp. 15-27.https://monoskop. org/images/6/66/Cross_ Nigel_1993_A_History_of_Design_Methodology.pdf

Doberti, R. (2006).Espacialidades, Buenos Aires:Infinito

Frayling, C. (1993). Research in art and design.EnRoyal College of Art Research Papers series No 1, Vol 1.London: Royal College of Art.

Fernández, R. (2013) Inteligencia proyectual Un manual de investigación en arquitectura Colección UAI - Investigación, Editorial Teseo, Buenos Aires 
Goodman, N. (1995).De la mente y otras materias, Madrid: Visor.

Hacking, I. (1996). Representar e intervenir, México D.F.: Paidós.

Martin Iglesias, R.; Motta, J. M.; Bohórquez Nates, M.; Speranza, F.; Turrillo, G.; Speziale, A. y Álvarez de Toledo, F. (2012). Hacia una epistemología del Diseño: construcción del conocimiento proyectual", ponencia presentada en XXVI Jornadas de Investigación y VIII Encuentro Regional SI + PI, Proyecto Integrar, SI - FADU - UBA

Platón (1871) El Banquete o del amor En Obras completas de Platón, tomo 5, Madrid: Medina y Navarro,

Sarquis, J. (2004). Itinerarios de proyecto. La investigación proyectual como forma de conocimiento en arquitectura. Buenos Aires: Nobuko.

Schon, D. (1998). La formación de Profesionales reflexivos. Madrid: Paidós.

\begin{abstract}
The purpose of this writing is to provide some clarifications about a possible epistemology of design, reflecting on the different approaches that an investigation can take in the field of the project.
\end{abstract}

Keywords: Epistemology of Design - research on Design - research for Design - research through Design - Project research.

Resumo: O objetivo deste escrito é fornecer alguns esclarecimentos sobre uma possível epistemologia do design, refletindo sobre as diferentes abordagens que uma investigação pode assumir no campo do projeto.

Palavras-chave: Epistemologia do Design - pesquisa em Design - pesquisa para Design pesquisa por meio do Design - pesquisa de projeto.

[Las traducciones de los abstracts fueron supervisadas por el autor de cada artículo] 